Check for updates

Cite this: Mater. Chem. Front., 2019, 3, 2775

DOI: 10.1039/c9qm90050k

rsc.li/frontiers-materials

\section{Correction: The design of Janus black phosphorus quantum dotsametal-organic nanoparticles for simultaneously enhancing environmental stability and photodynamic therapy efficiency}

\author{
Da Zhang, $\dagger^{\text {abc }}$ Ziguo Lin, $\dagger^{\text {acd }}$ Shanyou Lan, ${ }^{\text {acd }}$ Haiyan Sun, *e Yongyi Zeng ${ }^{* a b c d}$ and \\ Xiaolong Liu*abc
}

Correction for 'The design of Janus black phosphorus quantum dotsametal-organic nanoparticles for simultaneously enhancing environmental stability and photodynamic therapy efficiency' by Da Zhang et al., Mater. Chem. Front., 2019, 3, 656-663.

The authors regret that Fig. 6B in their article included an incorrect image. The H\&E staining image of lung tissue from the PBS/ laser group was incorrect. Fig. 6 with the correct staining image (second from top under 'Lung') is shown below.

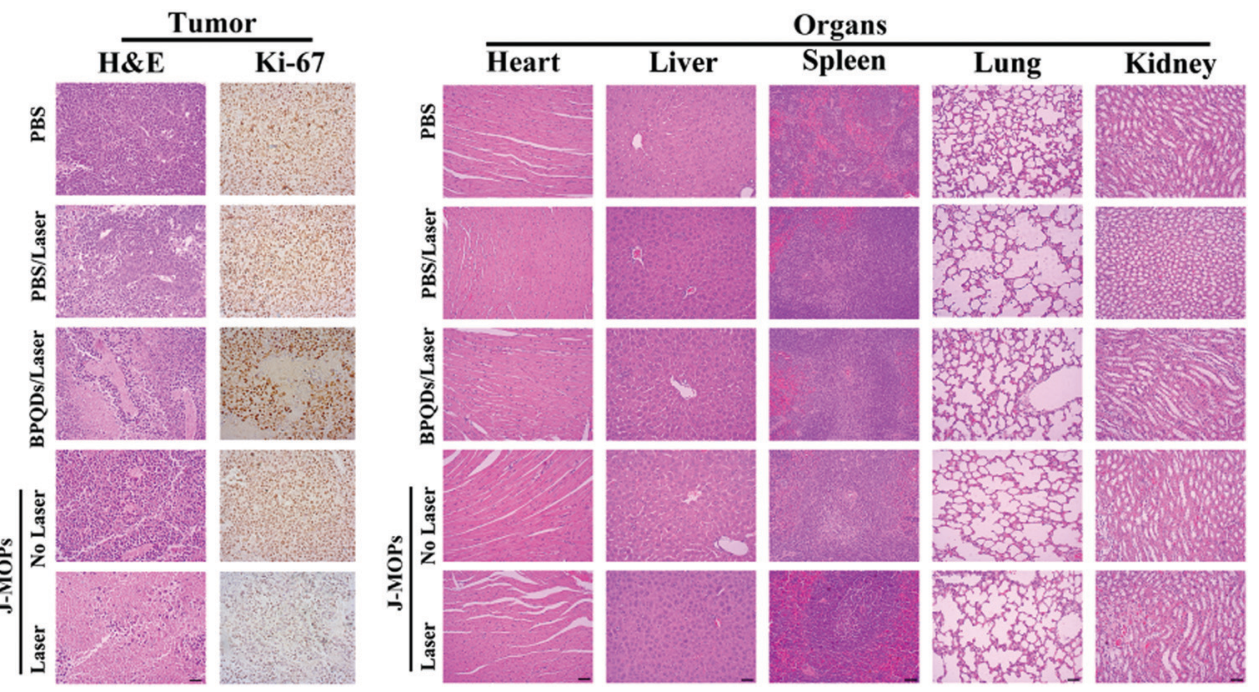

The Royal Society of Chemistry apologises for these errors and any consequent inconvenience to authors and readers.

\footnotetext{
${ }^{a}$ The United Innovation of Mengchao Hepatobiliary Technology Key Laboratory of Fujian Province, Mengchao Hepatobiliary Hospital of Fujian Medical University, Fuzhou 350025, P. R. China. E-mail: xiaoloong.liu@gmail.com

${ }^{b}$ Mengchao Med-X Center, Fuzhou University, Fuzhou 350116, P. R. China

${ }^{c}$ The Liver Center of Fujian Province, Fujian Medical University, Fuzhou 350025, P. R. China

${ }^{d}$ Liver Disease Center, The First Affiliated Hospital of Fujian Medical University, Fuzhou 350005, P. R. China. E-mail: lamp1973@medmail.com.cn

${ }^{e}$ Department of Anesthesiology, Beijing Anzhen Hospital, Capital Medical University, Beijing 100029, P. R. China. E-mail: 2254103187@qq.com

$\dagger$ These authors contributed equally to this work.
} 